\title{
Adaptação brasileira do Inventario de Autoeficacia para el Estudio
}

\author{
Patrícia Nunes da Fonsêca' \\ Universidade Federal da Paraíba - UFPB, PB, Brasil \\ Ricardo Neves Couto \\ Universidade Federal da Paraíba - UFPB, PB, Brasil \\ Maria Izabel Fernandes da Silva \\ Universidade Federal da Paraíba - UFPB, PB, Brasil \\ Gabriel Lins de Holanda Coelho \\ Cardiff University, Reino Unido \\ Thayro Andrade Carvalho
}

Universidade Federal do Rio Grande do Norte - UFRN, RN, Brasil

\begin{abstract}
Resumo: Este estudo tem como objetivo adaptar e validar o Inventario de Autoeficacia para el Estudio (Idape). Contou-se com 256 universitários, cuja média de idade foi de 22,96 anos $(D P=5,73)$, sendo $58,6 \%$ do sexo feminino. A maioria desses universitários foi proveniente de instituições particulares $(5 \mathrm{I}, 2 \%)$. Os participantes responderam ao Idape e a questões sociodemográficas. Por meio de análise fatorial exploratória, verificou-se que o Idape ficou composto por oito itens em um único fator, explicando $39 \%$ da variância total e apresentando alfa de Cronbach de 0,77 . Observou-se que a autoeficácia não diferiu em função do sexo, apresentando diferença somente quanto ao tipo de instituição. A quantidade de horas diárias de estudos e a autopercepção como estudante se correlacionaram positiva e significantemente com a autoeficácia. Conclui-se que o instrumento possui evidências psicométricas satisfatórias para investigar a autoeficácia e atividades autorreguladoras da aprendizagem no contexto brasileiro.
\end{abstract}

Palavras-chave: autoeficácia; estudantes; ensino superior; testes psicológicos; validade.

\section{BRAZILIAN ADAPTATION OF THE SELF-EFFICACY FOR STUDY INVENTORY INVENTARIO DE AUTOEFICACIA PARA EL ESTUDIO}

\begin{abstract}
This study aimed to adapt and validate the Inventario de Autoeficacia para el Estudio (Idape). Participants were 256 university students, with mean age of 22.96 $(S D=5.73)$ and $58.6 \%$ female. The majority of these came from private institutions (5I.2\%). Participants answered the Idape and sociodemographic questions. Performing an exploratory factor analysis, it was verified that the Idape was composed by eight items in one factor, explaining $39 \%$ of the total variance and presenting a Cronbach's alpha of .77. It was observed that the self-efficacy did not differ according to the sex, presenting differences regarding to the type of institution. The number of hours of study per day and self-perception as a student correlated positively and significantly with self-efficacy. It can be concluded that the instrument has satisfactory psychometric evidences to investigate self-efficacy and self-regulating activities of learning in the Brazilian context.
\end{abstract}

Keywords: self-efficacy; students; higher education; psychological tests; validity.

${ }^{1}$ Endereço de correspondência: Patrícia Nunes da Fonsêca: Universidade Federal da Paraíba, Centro de Educação, Departamento de Psicopedagogia, João Pessoa, PB, Brasil. CEP: 58051-900. E-mail: pnfonseca.ufpb@gmail.com 


\begin{abstract}
Resumen: Este estudio tiene objetivo adaptar y validar el Inventario de Autoeficacia para el Estudio (Idape). Se contó con 256 universitarios, cuya media de edad fue de 22,96 años $(D P=5,73)$, siendo $58,6 \%$ del sexo femenino. La mayoría fue proveniente de instituciones particulares $(5 \mathrm{I}, 2 \%)$. Los participantes respondieron el Idape y las cuestiones sociodemográficas. Por medio de análisis factorial exploratoria, verificó que el Idape estuvo compuesto por ocho artículos en un único factor, explicando $39 \%$ la variancia total y presentando alfa Cronbach 0,77 . Se observó que autoeficacia no difirió en función del sexo, presentando diferencia solamente cuanto al tipo de institución. La cantidad de horas diarias de estudios y autopercepción como estudiante se correlacionaron positivamente y significativamente con la autoeficacia. Concluye que el instrumento tiene evidencias psicométricas satisfactorias para investigar autoeficacia y actividades auto reguladoras del aprendizaje en el contexto brasileño.
\end{abstract}

Palabras clave: autoeficacia; estudiantes; educación superior; testes psicológicos; validez.

\title{
Introdução
}

O ingresso no ensino superior é um período que implica mudanças na vida dos estudantes. Nessa nova etapa, são constantes as situações em que o aluno tem de demonstrar responsabilidade, autonomia no processo de ensino-aprendizagem e bom desempenho acadêmico. Contudo, percebe-se nas escolas e universidades brasileiras o grande número de estudantes dependentes das orientações do professor para desenvolver atividades que poderiam realizar sozinhos, demonstrando pouca iniciativa (Rosa, 2014). Tal comportamento maximiza as dificuldades de os jovens se tornarem eficazes nos estudos e revela a necessidade de construir propostas de intervenção que auxiliem os estudantes a adotar estratégias de aprendizagem mais eficientes, administrar adequadamente o tempo e conquistar a autonomia no estudo (Costa \& Boruchovitch, 2010; Teixeira \& Alliprandini, 2013).

Diante desse cenário, constata-se a importância de conhecer melhor as variáveis que poderiam colaborar para o desenvolvimento integral do estudante, no sentido de gerar mais autoconfiança no seu processo de ensino-aprendizagem e, consequentemente, oferecer-Ihe melhor rendimento acadêmico. No presente estudo, selecionou-se a autoeficácia, caracterizada como um julgamento sobre as competências pessoais para a execução de uma ação voltada para um domínio específico (Bandura, 1997, 2005).

Segundo Bandura (2001), deve-se ressaltar que as pessoas são como produto e produtores de sistemas sociais, as quais, nas transições da vida, devem desenvolver crenças sobre suas capacidades para desempenhar determinadas atividades. Observa-se que a autoeficácia no âmbito educacional vem sendo apontada como um fator que instiga o estudante a se empenhar mais nos estudos e, assim, tornar-se mais persistente diante dos desafios acadêmicos, já que envolve a sua capacidade para se organizar e executar cursos de ações requeridas na produção de certas realizações (Polydoro \& Guerreiro-Casanova, 2010). Ademais, é um construto que tem recebido uma atenção especial, gerando importantes avanços de investigação no melhoramento de práticas pedagógicas e das performances educacionais (Sharma \& Nasa, 2014). 
Patrícia Nunes da Fonsêca, Ricardo Neves Couto, Maria Izabel Fernandes da Silva, Gabriel Lins de Holanda Coelho, Thayro Andrade Carvalho

Para Bandura (1993), o desenvolvimento da autoeficácia perpassa por quatro aspectos, a saber: 1) experiências diretas, que se referem às atividades realizadas com êxito, sendo estas influenciadas diretamente pelas informações positivas e negativas, que fortalecem a percepção acerca da crença; 2) experiências vicárias, os estudantes observam os modelos semelhantes a si mesmos, aprendendo novas estratégias, assim como percebem que possuem capacidade para exercerem tais papéis; 3) persuasão social, ocorre quando o ambiente social em que o estudante vive proporciona a ele a percepção de que é capaz de exercer tarefas difíceis, solucionar problemas e desenvolver atividades, e ele se esforçará mais para resolver determinadas situações; e 4) estados físicos e emocionais, como a ansiedade e o sono, pois, dependendo de como esses estados são interpretados, podem desencadear uma visão negativa ante determinadas resoluções de tarefas.

Especificamente na autoeficácia acadêmica, identificam-se dois componentes: 1) a expectativa de eficácia, aqui o estudante possui a convicção de que tem capacidade de gerar um resultado positivo, e 2) a expectativa de resultado, que é a crença de que o sujeito possui comportamentos que irão gerar um resultado especifico (Hall, Lindzey, \& Campbel, 2000).

Partindo dessa premissa, evidencia-se que as crenças de autoeficácia ajudam nas escolhas das atividades cotidianas e dos meios utilizados para alcançar os resultados esperados, mantendo o esforço e a persistência na tarefa. Atrelado a esse aspecto, o universitário, ao desempenhar o seu papel de forma bem-sucedida, sente-se mais confiante em si mesmo e tende a aumentar suas crenças do self e as expectativas perante os resultados, perseverando seus interesses de poder exercer a profissão almejada (Odaci, 2013).

Dessa maneira, a autoeficácia acadêmica está relacionada à aprendizagem, pois proporciona o desenvolvimento de ideias acerca de suas capacidades e competências, fazendo com que o estudante possa garantir a própria motivação para executar tarefas (Azzi, Guerreiro-Casanova, \& Dantas, 2014). Nesse caso, percebe-se que uma autopercepção positiva quanto ao potencial de execução serve como reforço para se empenhar e buscar bons resultados (por exemplo, sucesso acadêmico e autoestima) (Ornelas, Blanco, Gastélum, \& Chávez, 2012).

Um dos aspectos que fazem com que a crença de autoeficácia acadêmica se torne tão importante para a aprendizagem é o fato de ela não garantir a habilidade necessária para a efetivação de determinada tarefa com êxito. Entretanto, motiva o estudante a realizar suas ações com eficácia, levando ao desenvolvimento de habilidades e a melhoras na aprendizagem (Polydoro \& Guerreiro-Casanova, 2010).

Ademais, destacam-se cinco competências essenciais para uma aprendizagem eficaz, a saber: planejamento e gestão do tempo de estudo, compreensão de leitura, escrita expositiva, preparação para exames e anotações. Cada uma pode ser instruída por meio da autorregulação e, assim, gerar confiança no indivíduo a ponto de ele mesmo compreender que é capaz de realizar determinadas tarefas e identificar aquela que gera um impacto maior em sua vida (Masotti, 2014). 
As crenças de autoeficácia são referidas como fortes determinantes do nível de realização que os indivíduos podem alcançar. Não sendo surpresa, portanto, a forte associação positiva entre essa variável e a autorregulação da aprendizagem (Joly, Serpa, Borges, \& Martins, 2016), estratégias de aprendizagem (Dantas, Guerreiro-Casanova, Azzi, \& Benassi, 2015) e inteligência emocional, além de apresentar relação negativa com procrastinação acadêmica (Hen \& Goroshit, 2014).

Segundo Guerreiro-Casanova, Dantas e Azzi (2011), o contexto familiar pode influenciar no julgamento da autoeficácia dos estudantes, pois, nas relações cultivadas com os pais, os filhos podem desenvolver uma autopercepção acadêmica positiva ou negativa, a qual poderá interferir nas escolhas de atividades, no estabelecimento de metas, na quantidade de esforço despendido nas tarefas e na adequação ante a adversidade (Polydoro \& Guerreiro-Casanova, 2010). Karino e Laros (2014) verificaram que muitos jovens que enfrentam processos seletivos não se consideravam preparados física nem psicologicamente para realizar a avaliação, o que revela um baixo nível de autoeficácia.

Portanto, avalia-se que estudantes com crenças mais altas tendem a ser mais engajados nas atividades escolares e persistentes diante dos desafios advindos das tarefas acadêmicas, o que pode potencializar variáveis sociodemográficas. Corroborando essa perspectiva, Silva, Beltrame, Viana, Capistrano e Oliveira (2014) identificaram diferenças quanto à variável sexo, encontrando nível de autoeficácia estatisticamente superior para as meninas do que para os meninos. Contudo, Serpa, Soares e Fernandes (2015) asseveram que não existem diferenças no que concerne às atividades de autorregulação de estudo e às crenças de autoeficácia em relação ao sexo de estudantes.

Em síntese, conclui-se que a autorregulação é uma variável que exerce influência na escolha dos comportamentos que serão adotados como prática no dia a dia, passando a ser um elemento essencial no que se refere ao desenvolvimento pessoal, para as adaptações às situações de vida e às mudanças pessoais. Contudo, alguns a fazem de forma menos eficiente, podendo assim ocasionar comportamentos de evitação e dificuldades de execução das avaliações, em virtude da falta de segurança no modo como se prepararam para o exame (Masotti, 2014).

Isso posto, considera-se que é relevante mensurar a autoeficácia, sobretudo no contexto acadêmico, já que grande parte da população infantojuvenil vivencia situações de avaliação escolar diariamente. Na literatura, é possível encontrar estudos que se utilizam de instrumentos que avaliam a autoeficácia com atividades ocupacionais (Nunes \& Noronha, 2008), as funções de autoeficácia para escolha profissional (Ambiel \& Noronha, 2011) e a autoeficácia na formação superior (Polydoro \& Guerreiro-Casanova, 2010). No contexto acadêmico, destaca-se o uso do Inventario de Autoeficacia para el Estudio - Idape (Pérez \& Delgado, 2006).

Ressalta-se que o Idape é baseado na teoria social-cognitiva da aprendizagem e avalia estratégias de regulação. Originalmente, construído e validado em contexto argentino, foi aplicado a uma amostra de 291 adolescentes de escolas públicas e privadas, obtendo índices de validade e precisão satisfatórios. Dessa forma, ao avaliar a 
importância de medir esse construto na área acadêmica, este trabalho tem como objetivo adaptar e validar o Idape para o contexto brasileiro, com uma amostra de universitário, especificamente verificar a influência de variáveis sociodemográficas e correlacionar o Idape com as horas de estudo e a autopercepção como estudante.

\section{Método}

\section{Participantes}

Participaram 256 universitários das cidades de João Pessoa, Campina Grande e Patos (PB), com idade entre 18 e 55 anos $(M=22,96, D P=5,73)$. A maioria era do sexo feminino $(58,6 \%)$, proveniente de instituições particulares $(51,2 \%)$ e dos cursos de Psicopedagogia (18,2\%), Psicologia (17,2\%), Ciências da computação (15,9\%), Direito $(9 \%)$ e Nutrição $(8,2 \%)$. Esses universitários afirmaram estudar em média 2,53 horas por dia $(D P=1,81)$, e $54,7 \%$ se consideravam bons estudantes.

\section{Instrumentos}

- Inventario de Autoeficacia para el Estudio (Idape): proposto por Pérez e Delgado (2006), na língua espanhola, é composto por oito itens que mensuram as estratégias de aprendizagem e autorregulação - por exemplo, item 5: "Fazer perguntas a mim mesmo, para comprovar se eu compreendi um texto" (usando questionários ou manuais, por exemplo). É respondido em escala tipo Likert de 10 pontos, variando de 1 "Nada seguro" a 10 "Totalmente seguro" e apresenta evidências de validade e consistência interna $(\alpha=0,77)$.

- Questionário sociodemográfico: conjunto de perguntas - por exemplo, tipo de instituição, sexo, idade, curso, horas de estudo por dia, reprovação em disciplinas e autopercepção como estudante, avaliada em uma escala de 0 (péssimo) a 4 (ótimo) - que objetiva caracterizar os participantes, bem como fazer análises comparativas.

\section{Procedimento}

O presente estudo foi avaliado e aprovado pelo Comitê de Ética da Universidade Federal da Paraíba - Certificado de Apresentação para Apreciação Ética (CAAE) n. 47660615.1.0000.5183 -, conforme Parecer do Comitê de Ética em Pesquisa do Hospital Universitário Lauro Wanderley (CEP/HULW) n. 1798340. Inicialmente, realizou-se a tradução do instrumento do espanhol para o português. Para isso, contou-se com juízes experts e psicólogos pesquisadores bilíngues (português-espanhol) para analisar as versões traduzidas, seguido de uma tradução reversa para o espanhol (back-translation). As versões foram comparadas em termos de sua equivalência, comprovando-se que a tradução para o português refletia adequadamente o sentido da medida em espanhol. Finalmente, contou-se com 20 universitários, distribuídos equitativamente entre os sexos, e procedeu-se à validação semântica, na qual não foram identificadas necessidades de mudanças, dando prosseguimento à coleta de dados. 
A coleta ocorreu em instituições de ensino superior, públicas e privadas, seguindo os procedimentos éticos, conforme Resolução n. 466/12 do Conselho Nacional de Saúde. Os universitários foram convidados a participar da pesquisa de forma voluntária e informados de que poderiam desistir a qualquer momento, sem qualquer dano pessoal. A participação foi efetivada com a assinatura do Termo de Consentimento Livre e Esclarecido (TCLE). No momento da aplicação, foi explanado o objetivo geral da pesquisa, bem como o anonimato das respostas. Ressalta-se que a coleta de dados foi realizada em ambiente coletivo (sala de aula), mas os instrumentos foram respondidos de forma individual, apresentando em média 10 minutos para a conclusão.

\section{Análise dos dados}

Utilizaram-se o SPSS, em sua versão 23, para realização das estatísticas descritivas (média e desvio padrão) com a finalidade de caracterizar os participantes; a MANOVA, a fim de averiguar o poder discriminativo dos itens; e o teste $t$ para amostras independentes, objetivando verificar a influência do sexo e do tipo de instituição no índice de autoeficácia. Por fim, realizaram-se correlações $r$ de Pearson, com intervalos de confiança de $95 \%$ do coeficiente calculado a partir de bootstrap com 1.000 amostras, buscando a relação da autoeficácia com a percepção como estudante e horas de estudo diárias. Com o software Factor 9.2 foi possível investigar a dimensionalidade do Idape com o método Hull Comparative Fit Index, a partir de uma análise fatorial exploratória categórica Unweighted Least Squares (ULS), e checar a consistência interna por meio do alfa de Cronbach $(\alpha)$ com base nas correlações policóricas e a homogeneidade por meio da correlação média interitens.

\section{Resultados}

Inicialmente, procurou-se conhecer o poder discriminativo dos itens, formando dois grupos critérios, inferior e superior, a partir da mediana da pontuação total do Idape. Por meio de uma MANOVA, pode-se verificar que os itens discriminam pessoas com pontuações próximas. De fato, pessoas que pontuam baixo na escala pontuam baixo em cada um dos itens. Essa informação auxiliou na decisão pela permanência dos itens entre o conjunto que o compõe. Observou-se que todos os itens discriminaram na direção esperada (Lambda de Wilks $=0,32, F(8,247)=65,05, p<0,001, \eta^{2}=$ $0,68)$. Esses resultados podem ser verificados na Tabela 1.

Posteriormente, obteve-se a adequação da matriz de correlações policóricas para a realização da análise fatorial exploratória ordinal ULS, por meio dos índices satisfatórios do Kaiser-Meyer-Olkin (KMO) = 0,83 e do teste de esfericidade de Bartlett $=601,1(28)$; $p<0,01$. A análise de dimensionalidade pelo método Hull (Comparative Fit Index CFI) sugeriu uma solução unidimensional, resultando em um índice de ajuste Global Fit Index $(\mathrm{GFI})=0,99$. O fator retido (autovalor $=3,13$ ) explicou 39,14\% da variância total dos itens. As cargas fatoriais dos itens do Idape e a consistência interna da escala são apresentadas na Tabela 2. 


\section{Tabela I. Poder discriminativo dos itens do Idape.}

\begin{tabular}{|c|c|c|c|c|c|c|c|}
\hline \multirow[b]{3}{*}{ Itens } & \multicolumn{4}{|c|}{ Grupo critérios } & \multirow{2}{*}{\multicolumn{3}{|c|}{ Contraste }} \\
\hline & \multicolumn{2}{|c|}{ Inferior $(n=128)$} & \multicolumn{2}{|c|}{ Superior $(n=128)$} & & & \\
\hline & M & $D P$ & $M$ & $D P$ & $F$ & $p$ & $\eta^{2} p$ \\
\hline 1 & 4,75 & 0,19 & 7,07 & 0,19 & 68,55 & $0,001 *$ & 0,21 \\
\hline 2 & 4,51 & 0,19 & 7,73 & 0,19 & $|39,3|$ & $0,001 *$ & 0,35 \\
\hline 3 & 6,16 & 0,14 & 8,58 & 0,14 & 150,43 & $0,001 *$ & 0,37 \\
\hline 4 & 5,88 & 0,18 & 7,96 & 0,18 & 61,15 & $0,001 *$ & 0,19 \\
\hline 5 & 6,24 & 0,18 & 8,53 & 0,18 & 80,80 & $0,001 *$ & 0,24 \\
\hline 6 & 5,75 & 0,17 & 7,99 & 0,17 & 80,95 & $0,001 *$ & 0,24 \\
\hline 7 & 4,73 & 0,19 & 7,05 & 0,19 & 69,81 & $0,001 *$ & 0,22 \\
\hline 8 & 5,79 & 0,18 & 8,08 & 0,18 & 75,40 & $0,001 *$ & 0,23 \\
\hline
\end{tabular}

Nota: * Item discriminativo $(p<0,0 \mathrm{I})$.

Tabela 2. Estrutura fatorial da Idape.

Itens

03. Fixar-me em objetivos escolares (obter notas altas, por exemplo) e esforçar-me para alcançá-los.

05. Fazer perguntas a mim mesmo, para comprovar se eu compreendi um texto (usando questionários ou manuais, por exemplo).

06. Relacionar os conceitos novos, que estou estudando, com outros que já conheço (fazendo um esquema, por exemplo).

08. Dedicar mais horas ao estudo, do que havia planejado (quando se aproxima a prova, por exemplo).

04. Fazer os deveres mesmo tendo coisas mais atrativas para realizar (sair com os meus amigos, por exemplo).

02. Perguntar na aula quando não compreendo a explicação do professor.

07. Planejar uma redação extensa ou um relatório antes de escrever (fazendo uma lista dos principais temas a serem desenvolvidos).

0I. Assistir à aula mesmo que tenha alguma preocupação naquele momento.

0I. Assistir à aula mesmo q
Quantidade de itens

Carga fatorial $h^{2}$

$0,69 *$

0,47

$0,61 *$

$0,59 *$

0,35

0,56 *

$0,54 *$

0,5 I*

0,26

$0,49 *$

Variância explicada (\%)

39,14

Valor próprio

3,13

Alfa de Cronbach

Nota: * Carga fatorial considerada satisfatória, isto é, $>|0,40| ; h^{2}=$ comunalidade.: 
Observa-se que o fator geral, denominado autoeficácia acadêmica, apresentou valor próprio de 3,12 e ficou composto por todos os itens com cargas variando de 0,41 (item 01: "Assistir à aula mesmo que tenha alguma preocupação naquele momento") a 0,69 (item 03: "Fixar-me em objetivos escolares (obter notas altas, por exemplo) e esforçar-me para alcançá-los"). Ademais, apresentou um alfa de Cronbach com base em correlações policóricas $(0,77)$ e a homogeneidade média dos itens de 0,22 (correlação média interitens; rm.i), demonstrando que a escala apresenta consistência interna satisfatória. Portanto, esses resultados apresentam evidências psicométricas satisfatória do instrumento para seu uso no Brasil.

\section{Influência das variáveis demográficas}

Posteriormente, foram realizadas análises acerca das fontes de variações no índice geral de autoeficácia acadêmica, calculado a partir do somatório de todos os itens. Buscando fazer comparações, realizou-se o teste $t$ de Student para amostras independentes com duas variáveis sociodemográficas dos participantes (sexo e tipo de instituição). Os resultados revelaram que não havia diferença significativa das médias quanto ao sexo $(t(253)=1,09 ; p=0,27)$ dos universitários. Entretanto, encontrou-se uma diferença significativa das médias quanto ao tipo de instituição $(t(254)=2,63 ; p<0,01$; $d=0,33)$, sugerindo que os universitários oriundos de instituições particulares $(M=$ $55,31 ; D P=12,17)$ apresentam maiores índices de autoeficácia em relação aos da pública $(M=51,45 ; D P=11,19)$.

Dando continuidade às análises, foram executadas correlações $r$ de Pearson a fim de buscar o grau de relação entre o índice de autoeficácia dos universitários e a quantidade de horas de estudos destinadas por dia, e a autopercepção como estudante. Os resultados demonstram correlações positivas e estatisticamente significativas tanto com as horas de estudos $(r=0,16$; IC $95 \%=0,05 / 0,38 ; p<0,01)$ quanto com a autopercepção como estudante $(r=0,35$; IC $95 \%=0,22 / 0,46 ; p<0,001)$. Destarte, os resultados sugerem que altos índices de autoeficácia acadêmica são relacionados com uma maior carga horária de estudos e uma autopercepção mais positiva sobre sua vida acadêmica.

\section{Discussão}

Este artigo objetivou reunir evidências de adequação psicométrica do Idape considerando sua validade fatorial e consistência interna. Confia-se que esse objetivo tenha sido alcançado. Após as análises realizadas, o Idape ficou composto por todos os oito itens, assim como na estrutura encontrada na Argentina, em seu estudo original (Perez \& Delgado, 2006). Os itens tiveram saturação mínima igual ou superior a $|0,40|$ e foram distribuídos explicando $39,01 \%$ da variância total, apresentando também índices de precisão satisfatórios - alfa de Cronbach e homogeneidade média dos itens (Cohen, Swerdlik, \& Sturman, 2014). O modelo unifatorial corrobora os achados originais e a operacionalização da autoeficácia voltada para um domínio específico (Bandura, 1997, 2005). 
Em seguida, neste estudo, não foi encontrada diferença significativa do índice de autoeficácia quanto ao sexo dos universitários, corroborando assim com os achados de Serpa et al. (2015). Esses autores sugerem que homens e mulheres não diferem quanto à sua programação e às ações para o estudo. Quanto ao tipo de instituição, observou-se que os universitários de instituições particulares apresentam maiores índices de autoeficácia acadêmica. A partir dessa distinção, chama-se a atenção para o contexto social, tais como a pressão por parte dos familiares que dispõem de um valor pecuniário pelo ensino e, portanto, exigem que o estudante se dedique o máximo aos estudos, fato que o instiga a se organizar em termos de estratégias de aprendizagem para apresentar um bom desempenho acadêmico (Serpa et al., 2015).

Ademais, verificou-se que maiores horas de estudo por dia e uma elevada autopercepção como estudante se relacionam positivamente com autoeficácia acadêmica, indicando que os universitários enxergam a necessidade de desenvolver a própria motivação para realizar suas tarefas e, portanto, buscam aumentar seu tempo destinado para o estudo (Azzi et al., 2014). É provável que, ao se perceber como capaz de executar seus deveres como aprendiz, obtém melhores resultados e, consequentemente, uma maior autoestima e autoeficácia acadêmica (Vega, Contreras, Chávez, \& Delgado, 2012).

Em síntese, com base nos resultados, conclui-se que tais objetivos foram alcançados e podem ser encarados como confiáveis. Contudo, apesar de os objetivos propostos terem sido atingidos, contribuindo para os achados da literatura especializada, este estudo apresenta como limitação o caráter da amostra, por conveniência. Não podendo generalizar os resultados nem para a população da qual a amostra foi extraída. Outrossim, vale ressaltar que esse ponto não fazia parte do objetivo da pesquisa.

\section{Considerações finais}

Em estudos posteriores, sugere-se a utilização de amostras maiores e mais diversificadas, compostas por participantes das diferentes regiões do Brasil. Também se recomenda a realização de análises mais robustas (por exemplo, análise fatorial confirmatória e invariância quanto ao sexo ou tipo de instituição), a fim de refinar o uso do instrumento e buscar a relação com outras variáveis (por exemplo, ansiedade ante o exame, engajamento escolar e desempenho acadêmico), reunindo evidências de validade externa.

Conclui-se que os resultados atestam a possibilidade de aplicação da versão brasileira do Idape no processo de avaliação da autoeficácia dos estudantes, incluindo itens que mencionam estratégias autorreguladoras de aprendizagem (Pérez \& Delgado, 2006). Dessa forma, pode-se estimular o desenvolvimento de novas técnicas na gestão acadêmica, assim como direcionar novas práticas docentes e incentivar intervenções no ensino superior. 


\section{Referências}

Ambiel, R. A. M., \& Noronha, A. P. P. (2011). Construção dos itens da escala de autoeficácia para escolha profissional. Psico-USF, 16(1), 23-32. doi:10.1590/ S1413-82712011000100004

Azzi, R. G., Guerreiro-Casanova, D. C., \& Dantas, M. A. (2014). Autoeficácia acadêmica: percepções de estudantes brasileiros. In R. G. Azzi \& D. A. Vieira (Orgs.), Crenças de eficácia em contexto educativo (pp. 67-83). São Paulo: Casa do Psicólogo.

Bandura, A. (1993). Perceived self-efficacy in cognitive development and functioning. Educational Psychologist, 28, 117-148. Recuperado em 11 outubro, 2018, de https://www.uky.edu/ eushe2/Bandura/Bandura1993EP.pdf

Bandura, A. (1997). Self-efficacy, the exercise of control. New York: Freeman and Company.

Bandura, A. (2001). Social cognitive theory: an agentic perspective. Annual Reviews Psychologist, 52(1), 2-18. doi:10.1146/annurev.psych.52.1.1

Bandura, A. (2005). Guide for constructing self-efficacy scales. In A. Brandura, Self-efficay beliefs of adolescents (pp. 307-337). Recuperado em 11 outubro, 2018, de https://www.uky.edu/ eushe2/Bandura/BanduraGuide2006.pdf

Cohen, R. J., Swerdlik, M. E., \& Sturman, E. D. (2014). Testagem e avaliação psicológica: introdução a testes e medidas (8a. ed.) São Paulo: AMGH.

Costa, E. R., \& Boruchovitch, E. R. (2010). As estratégias de aprendizagem de alunos repetentes do ensino fundamental. Psicologia em Pesquisa, 4(1), 31-39. Recuperado em 11 outubro, 2018, de http://pepsic.bvsalud.org/pdf/psipesq/v4n1/ v4n1a05.pdf

Dantas, M. A., Guerreiro-Casanova, D. C., Azzi, R. G., \& Benassi, M. T. (2015). Relação entre autoeficácia acadêmica e estratégias de estudo e aprendizagem: mudanças ao longo do primeiro semestre do ensino médio. Psicologia: Ensino \& Formação, 6(1), 33-51. Recuperado em 11 outubro, 2018, de http://pepsic.bvsalud. org/scielo.php?pid=\$2177-20612015000100004\&script=sci_abstract

Guerreiro-Casanova, D. C., Dantas, M. A., \& Azzi, R. G. (2011). Autoeficácia de alunos do ensino médio e nível de escolaridade dos pais. Estudos Interdisciplinares em Psicologia, 2(1), 36-55. doi:10.5433/2236-6407.2011v2n1p36

Hall, S. C., Lindzey, G., \& Campbel, J. B. (2000). Teorias da personalidade (4a. ed.). Porto Alegre: Artmed.

Hen, M., \& Goroshit, M. (2014). Academic procrastination, emotional intelligence, academic self-efficacy, and GPA: a comparison between students with and without learning disabilities. Journal of Learning Disabilities, 47(2), 116-124. doi:0.1177/ 0022219412439325 
Joly, M. C. R. A., Serpa, A. L. O., Borges, L., \& Martins, R. M. M. (2016). Autoeficácia acadêmica e autorregulação da aprendizagem: rede de relacionamento em base online. Avaliação Psicológica, 15(1), 73-82. doi:10.15689/ap.2016.1501.08

Karino, C, A., \& Laros, J. A. (2014). Ansiedade em situações de prova: evidências de validade de duas escalas. Psico-USF, 19(1), 23-36. doi:10.1590/S1413-82712014000 100004

Masotti, D. R. (2014). Autoeficácia e autorregulação acadêmica contribuindo para a previsão da evasão escolar. Tear: Revista de educação, 3(2), 1-17. Recuperado em 28 de Outubro, 2018, de https://periodicos.ifrs.edu.br/index.php/tear/article/ view/1857/1441

Nunes, M. F. O., \& Noronha, A. P. P. (2008). Escala de auto-eficácia para atividades ocupacionais: construção e estudos exploratórios. Paidéia, 18(39), 111-124. Recuperado em 11 outubro, 2018, de http://www.scielo.br/pdf/paideia/v18n39/v18 n39a11.pdf

Odaci, H. (2013). Risk-taking behavior and academic self-efficacy as variables accounting for problematic internet use in adolescent university students. Children and Youth Services Review, 35(1), 183-187. doi:10.1016/j.childyouth.2012.09.011

Ornelas, M., Blanco, H., Gastélum, G., \& Chávez, A. (2012). Autoeficacia percibida en la conducta académica de estudiantes universitarias. Formación universitaria, 5(2), 17-26. doi:10.4067/S0718-50062012000200003

Pérez, E., R., \& Delgado, M. F. (2006). Inventario de autoeficacia para el estudio: desarrollo y validacion inicial. Avaliação Psicológica, 5(2), 135-143. Recuperado em 11 outubro, 2018, de http://www.redalyc.org/articulo.oa?id=335027180003

Polydoro, S. A. J., \& Guerreiro-Casanova, D. C. (2010). Escala de auto-eficácia na formação superior: construção e estudo de validação. Avaliação Psicológica, 9(2), 267-278. Recuperado em 11 outubro, 2018, de http://pepsic.bvsalud.org/scielo. php?script=sci_arttext\&pid=S1677-04712010000200011

Rosa, C. M. (2014). Limites da democratização da educação superior: entraves na permanência e a evasão na Universidade Federal de Goiás. Poíesis Pedagógica, 12(1), 240-257. doi:10.5216/rpp.v12i1.31219

Serpa, A. L. O., Soares, T. F., \& Fernandes, N. S. (2015). Variáveis do contexto escolar como preditoras da autoeficácia e ansiedade de estudantes. Avaliação Psicológica, 14(2), 189-197. doi:10.15689/ap.2015.1402.03

Sharma, H. L., \& Nasa, G. (2014). Academic self-efficacy: a reliable predictor of educational performances. British Journal of Education, 2(3), 57-64. Recuperado em 11 outubro, 2018, de http://www.eajournals.org/wp-content/uploads/ Academic-Self-Efficacy-A-Reliable-Predictor-of-Educational-Performances1.pdf 
Silva, J. da, Beltrame, T. S., Viana, M. da S., Capistrano, R., \& Oliveira, A. do V. P. de (2014). Autoeficácia e desempenho escolar de alunos do ensino fundamental. Revista Quadrimestral da Associação Brasileira de Psicologia Escolar e Educacional, 18(3), 411-420. doi:10.159/2175-3539/2014/0183760

Teixeira, A. R., \& Alliprandini, P. M. Z. (2013). Intervenção no uso de estratégias de aprendizagem diante de dificuldades de aprendizagem. Revista Semestral da Associação Brasileira de Psicologia Escolar e Educacional, 17(2), 279-288. Recuperado em 11 outubro, 2018, de http://www.scielo.br/pdf/pee/v17n2/v17n2a10.pdf

Vega, H. B., Contreras, M. O., Chávez, J. F. A., \& Delgado, J. C. G. (2012). Autoeficacia percibida en conductas académicas: diferencias entre hombres y mujeres. Revista Mexicana de Investigación Educativa, 17(53), 557-571. Recuperado em 11 outubro, 2018, de http://www.scielo.org.mx/scielo.php?script=sci_arttext\&pid= S1405-66662012000200011

O presente artigo contou com apoio do Conselho Nacional de Desenvolvimento Científico e Tecnológico (CNPq) e da Coordenação de Aperfeiçoamento de Pessoal de Nível Superior (Capes) por meio de financiamento do Projeto Universal do primeiro autor (CNPq), além de bolsa de mestrado ao segundo (Capes). Aproveitamos para demonstrar nossa gratidão a essas instituições.

Submissão: 2.12.2016

Aceite: 16.6.2018 